\title{
Building heritage communities online: The experience of the Summer School Program 'Engaging Communities in Cultural Heritage'
}

\author{
Claudia URIBE CHINEN \\ University of Tsukuba \\ curibec@pucp.pe
}

\section{Introduction}

The summer school program entitled 'Engaging Communities in Cultural Heritage' was held from 21 June to 5 July 2020, as part of the Executive Leadership Development in Heritage Management Workshops organized yearly by the Heritage Management Organization. This year in particular, the course was held entirely online due to the ongoing pandemic and subsequent worldwide responses that enforced preventive measures and social distancing. Amid these challenging circumstances, the 2-week program was successfully conducted, taking advantage of digital resources to materialize a syllabus designed to combine theoretical and practical perspectives regarding community engagement in heritage projects in Greece. The series of online meetings gathered 13 students from six countries in total (Greece, Iran, Kenya, Peru, Puerto Rico and Serbia) with different backgrounds and career experiences. This diversity enriched the dynamics of the workshop, where group discussions and assignments played a substantial role, and fostered a unique and fertile environment of intercultural and multidisciplinary work.

The relevance of capacity-building in heritage and community engagement is more than evident in the current global context of the pandemic. Firstly, it is necessary to raise awareness of local issues, but also to understand the varieties of experiences in other places in the world to gain a broader perspective that can lead to better-informed decisions. Secondly, the hiatus represented by the current lack of mass tourism and development projects is a critical moment to reflect on and rethink common heritage management issues, some of which originate in the

\section{AP: Online Journal in Public Archaeology - Volume 11 - ISSN: 2171-6315}

Uribe Chinen, C., J. 2021. Building heritage communities online: The experience of the Summer School Program 'Engaging Communities in Cultural Heritage'. AP: Online Journal in Public Archaeology 11, y7-y14.

DOI: 10.23914/ap.v11i0.284 


\section{y8 :: AP Journal v.11 :: POINTS OF YOU}

inequalities stemming from structural deficiencies, such as the over-centralization of heritage in the hands of the state, governmental agencies or experts, and the exclusion of community participation. The program of the workshop favoured spaces of encounter with Greek cultural heritage and community engagement projects, particularly the Paros Festival on the Greek island of Paros, and offered students diverse opportunities to learn, discuss and think creatively together with professors and persons involved in such heritage-related initiatives.

\section{The 2020 Workshop: form and contents}

The workshop was composed of seven meetings of two-to-three hours (starting at 15:00 Greek time) held on the Zoom online platform. Each of these meetings comprised lectures on core topics given by professors: Evangelos Kyriakidis, Aris Anagnostopoulos, Lena Stefanou and Katerina Konstantinou, from the Heritage Management Organization, alongside reflections, group activities and presentations of assignments, with logistic support continuously provided by Ms Fotini Giannoulidi. Despite the challenges of conducting lectures and activities in a non-physical, purely virtual environment, the workshop benefited from the possibilities of working synchronically and asynchronically with digital tools. The meetings were held with the simultaneous participation of lecturers and students, with the possibility of screen-sharing enabled to display materials, as would be the case in physical class attendance. Students were able to discuss and exchange opinions when interventions were allowed, and to work in groups in Zoom's breakout rooms or Google Docs in a way that could equate to face-to-face interaction to a certain degree. The fact that a 'chat box' was available in the online platform helped the questions and answers to be organized in a manner that would not interrupt the flow of the class. This particular aspect engaged professors and students in an interactive way; questions posed by students in the chat box were answered as soon as the lecturer found a space for them, and many ideas were exchanged in this process.

The program of the summer school was comprehensive and provided a fertile ground for learning a variety of topics, approaches and tools that are essential for working in community engagement projects and for problematizing the contexts limiting the participation of communities in cultural heritage management. Each of the lectures presented the topics of community engagement, ethnographic methods, oral history, and digitization respectively, and shared a consistent corpus of theory and praxis: not only did they explore theoretical and methodological frameworks, but they also introduced professors' experiences with various forms of relationships between people and heritage through their research programs, art projects and museum curatorship. A particular case study that allowed 
students to understand key concepts, assess context-based community engagement processes and develop activities for the workshop was the Paros Festival. This is a three-day cultural event celebrated every year on the Greek island of Paros, where local communities participate and engage in a variety of activities to explore and interact with the island's cultural values embodied in its tangible and intangible heritage.

Thanks to this structure, students could grasp fundamental concepts and reflect on the effectiveness, advantages, successes and ethical principles of certain tools when working with communities. Besides the online meetings with professors, students could access bibliographic references prepared by the organization on the Moodle platform, as well as the workshop's recorded lectures to reinforce the learning sessions. The workshop also represented an opportunity to foster skills central to an executive education, such as teamwork, communication, leadership, and innovation.

\section{The perspective gained through the lens of Community Engagement and the role of Ethnography}

Community engagement represents a means by which to democratize heritage and foment communities' abilities to have not only a say but also a central role in the management of their heritage; therefore, it paves the way for a more inclusive, multivocal and bottom-up approach to heritage. In the first lectures of the workshop, the principles of community engagement were explored through theoretical and practical perspectives. Understanding these principles is crucial to identifying the aspects that may become problematic in cultural heritage management projects, especially in those where participatory processes appear as a mandate of pre-established top-down models. In some cases, these projects assume communities to be agents by which standard procedures of management are fulfilled, or moreover, as a monolithic segment that will take responsibility for (their own) heritage while adopting imposed regulations. The lectures and assignments of the workshop allowed students to understand the diversity and complexity of communities as dynamic, context-dependent and variable agents, as well as to look into the key processes for identifying and working with them. It was emphasized that, since the varieties of communities' needs, priorities and expectations influence their views towards heritage or heritage management projects, it is important to consider that engagement should not be taken as a given, but instead it has to be worked toward and negotiated through an oftentimes long process. The adoption of community engagement, therefore, challenges the notion that communities blindly and passively assimilate heritage conservation frameworks. Such an engagement recognizes the divergent opinions and perspectives that 


\section{y10 :: AP Journal v.11 :: POINTS OF YOU}

may not necessarily be aligned with heritage official discourses. Conversely, it offers a chance to build heritage management projects from real dialogue, consensus, and the dynamism of communities themselves, while avoiding exclusion and disenfranchisement. Furthermore, it represents an opportunity for communities to be actively involved in the different stages of heritage management, as in some cases they are legally impeded from participating in decision-making processes in the elaboration of heritage management plans.

The applicability of community engagement in cultural heritage management finds a necessary ally in ethnographic methods. In the early stages of the workshop, ethnography was introduced as an essential tool by which to approach and involve communities in cultural heritage projects given its principles based on an immersion in the community itself in order to observe, study and understand behaviours and points-of-view. Based on a hypothetical mapping of communities involved in the Paros Festival, students were able to learn the importance of audience segmentation to organizing and systematizing the population to be addressed for ethnographic research. Further insights were gained from the outcomes of projects conducted in Greece by the professors. A highly important lesson from this methodological approach was the pivotal role ethnographic research plays in giving invisible or marginalized voices a chance to be heard. Thus, ethnographic research for community engagement results in two major changes to the conventional way of managing heritage: (1) experts decentralize their role and authority in the interpretation, communication and management of heritage values, and (2) local communities are assumed to also be experts, repositioning them from stakeholders who have to listen to the main stakeholders who have to be listened to. In this process, heritage can serve to reflect on what truly matters and is important to communities, and also serve to enable spaces for co-creation and synergies for mutual benefits.

\section{Digging into personal stories: the power of oral history}

Alongside community engagement and ethnography, oral history also constitutes a means for the fostering of bottom-up approaches to heritage and for overcoming a monolithic view of the past and its material remains. When working in heritage or archaeology projects, experts usually position themselves on the side of an official discourse, which, besides silencing and excluding alternative visions from communities or non-official actors, disguises the multiple grounds of encounters, contestations or even conflicts in the heritage process. Oral history is not the antidote, but rather a chance to de-centre from official discourses by hearing the voices of actors from the local level and giving them roles as authors of history themselves. It also provides insights into how heritage, its values, and its 


\section{:: Uribe Chinen - Building heritage communities online :: y11}

narratives, are entangled with memory and identity. The testimonies of oral history, together with their affective and emotional dimensions, are highly valuable to understanding history at a distance from the hegemonic perspective and from angles of very personal experiences.

Within the group activities, students explored the multiple layers of history and social processes intertwined in personal memories in an assignment that required the use of personal photographs. In another assignment, after being instructed on the principles of oral history and guidelines for interview questions, students were put into three groups to conduct interviews with three local residents of the island of Paros. As introduced in the lectures, any study based on ethnographic or oral story works essentially depends on the ways the researcher strategically defines their methods of entering a community and gaining access to informants. In this sense, and in order to follow a respectful approach as outsiders, relying on the support of a gatekeeper that helps the researcher to connect with informants is essentially an unavoidable step. This process was facilitated by the professors, who acted as gatekeepers for the students, and aided in the selection of the three interviewees, based on their networks and knowledge of their active involvement in the local cultural life and, more particularly, in the Paros Festival itself. The individuals who collaborated this time as interviewees were as follows: Mr Jeffrey Carson, a local promoter of arts and culture who has been based on Paros for over 50 years; Dimitra Skandali, a contemporary artist born and raised on Paros; and Monique Mailloux, a ceramic artist based on Paros. The teams prepared their own questions, taking into consideration the profiles of each of the interviewees and the ways their personal stories could be intertwined with the heritage values of Paros.

The interviews were conducted via the Zoom platform upon three different days assigned for each of the teams. Students could interact with the interviewees, asking questions in turns and developing wider conversations. Despite the lack of in-person interaction with the interviewees, the process went smoothly, and the questions were enthusiastically answered. One of the interviewees, $\mathrm{Mr}$ Jeffrey Carson, who is a US citizen permanently based on Paros, shared his memories of his arrival, engagement in the local cultural life and intellectual works in Greece. Through testimonies like this, students could read different layers of history, as well as the values and meanings of places, landscapes and practices for people living on the island. Furthermore, in getting to know these values, it was also possible to understand the preoccupations of local residents like Mr Carson regarding the impacts of mass tourism on local heritage and identity. Following the interviews, there was a session for the presentation of results and discussions on the technical aspects, considerations and challenges of video-recorded interviews. 


\section{y12 :: AP Journal v.11 :: POINTS OF YOU}

\section{The relevance of digital resources in the current context}

The current context of the global pandemic is a critical moment to reflect on the inconsistencies of the modus operandi, as well as to reinvent habitual protocols, practices and tools. In this workshop, a particular locus of reflection was the relevance of the use of digital and multimedia resources for arts and heritage projects. In the past decade, digitization of heritage, tangible and intangible alike, has been an important initiative undertaken by museums, cultural institutions, research centres and similar in order to not only secure the documentation of places, collections and traditions but also to increase their public accessibility, exploration, outreach and awareness. Amid the pandemic, as many activities are migrating to virtual spaces, the continuity and sustainability of projects would be highly reliant on the use of digital tools.

The lecture about the digital world placed an emphasis on the ways in which heritage can be presented and experienced through digital resources. In one assignment, students worked on the design of a digital application where the history of tourism in Paros could be introduced to visitors and to participants in the Paros Festival. An important aspect to this activity was the user-friendliness of the application with regard to the effective navigation through the contents and the legibility of the story being told. In another assignment, participants reflected on how the Paros Festival could work on digital world alternatives to engage the public that follows the activities of this event every year. Moreover, students were also invited to co-design the structure and contents of a podcast of the Paros Festival based on the interviews conducted as part of the session focused on oral history. In the podcast activity, not only was it possible to think about how the history of Paros could be presented through local and foreign eyes, but also how the narratives of the heritage values of the island could be conveyed.

\section{Results of the workshop}

The two-week program provided an enriching space for learning through lectures, discussions, exchange of ideas, collaborative work and creativity with a vibrant community of professors and students. Throughout meetings, where the mentoring role played by professors was crucial, students could gain a solid corpus of knowledge and skills to work on communities' engagement in heritage management projects. They were able to learn to distinguish, address and observe communities using ethnographic methods, understand the value of contributions of oral history, and expand their knowledge of the benefits of the use of virtual spaces and resources. Furthermore, each of the assignments worked as a laboratory of ideas, where students used their creativity and backgrounds in the co-design of hypothetical activities and resources for communities of locals 
on and visitors to Paros. The module on oral history and the interview sessions allowed students to compare and contrast the multiple ways in which historical processes, experiences and places are internalized in memory, finding similarities and divergences in the testimonies collected. Despite being virtual interactions, these interviews emulated the real fieldwork involved in an oral history project; therefore, they gave a glimpse of what forms ethnographic research may take in the 'new normal'. Finally, through collaborative work, the summer school's community of professors and students delivered a podcast for the Paros Festival, into which contents from the interviews were integrated to narrate how the local and the global interact on Paros to create a unique artistic landscape.

\section{Conclusion}

The topics explored in the workshop were geared towards a transformative practice in heritage management, where communities - or people in general - lie at the centre as opposed to the periphery when thinking about heritage research, conservation, interpretation and presentation. Communities are not to be included in heritage management projects solely due to official mandatory requirements, but rather because they are at the heart of building narratives, memories and identities attached to things, places, practices and landscapes of value to themselves, and these are fundamental aspects in strengthening their sense of place and well-being. Community engagement projects, ethnographic research and oral history can act as means to challenge the hierarchical and authoritative perspectives and frameworks that hinder the involvement of communities in heritage processes due to such processes' failure to consider their voices. By giving voices to communities, their fluidity, dynamism and even contradictions are also recognized; therefore, it is possible to build a broader range of context-based heritage management models that see heritage not in a fixed and agreed sense, invariable in time and space, but as a space for negotiation.

The theoretical, methodological and practical tools provided by the program pave the way for a major commitment in ethical and adaptive responses to current global issues. The importance of working with communities and giving them voices is understood not only because of the possibility for diversifying the narratives of heritage, but, more importantly, for materializing social justice in heritage practice. In a similar way, community engagement projects are venues for decolonizing heritage and communities themselves: heritage is understood in terms of its multi- temporality, and as a space where communities have a say and can be heard as authors of history, as opposed to a phenomenon monopolized by 'experts' in the field. Furthermore, in the context of the current pandemic, where multiple responses are emerging creatively to ensure the continuity of projects 


\section{y14 :: AP Journal v.11 :: POINTS OF YOU}

in the fields of culture, heritage and arts, most initiatives have begun to gravitate around the digital world. In general, our world at present demands large-scale awareness regarding the uses and advantages of virtual spaces and multimedia. The lessons learned in this workshop encourage participants to think about more ways to enrich the relationship between communities and heritage through digital resources in the 'new normal' and the post-COVID world.

\section{Personal reflection}

I would like to use this section to express my sincere gratitude to the Heritage Management Organization, its professors and members for giving us the chance to strengthen our capacities in heritage management with a solid commitment to socially engaged practice. Being trained in archaeology, but primarily in the positivist tradition, I constantly questioned myself as to the extent to which I attempted to grow my awareness of the social, reflexive and ethical dimensions of the discipline. In this process, I encountered barriers in the form of conceptions such as the prevalence of objectivity in archaeology and archaeology's engagement with social justice being limited to forensic archaeology projects dealing with cases of conflict, memory and human rights. For my graduate studies, I decided to enter the field of heritage studies with the aim of broadening my perspective and learning new approaches from which to understand the past, its material traces and how these relate to present-day societies. By focusing on 'heritage', or, more specifically, on the tangible and intangible embodiments of heritage values, my work would necessarily address the social dimension of the material past in contemporary times. I am more than grateful for the wonderful knowledge and lessons learned in this workshop; they will not only open new gates and expand horizons in my future endeavours, but also motivate me to look once again at past potential projects that were never implemented due to a lack of adequate perspective, which can now be developed from a fresh point of view. 